\title{
Necessary Conditions for Isolation of Special Classes of Bilinear Autoregressive Moving Average Vector (BARMAV) Models
}

\author{
Anthony Effiong Usoro*, Eyo Awakessien Clement \\ Department of Mathematics and Statistics, Akwa Ibom State University, Mkpat Enin, Nigeria \\ Email address: \\ anthonyusoro@aksu.edu.ng (A. E. Usoro) \\ ${ }^{*}$ Corresponding author \\ To cite this article: \\ Anthony Effiong Usoro, Eyo Awakessien Clement. Necessary Conditions for Isolation of Special Classes of Bilinear Autoregressive Moving \\ Average Vector (BARMAV) Models. American Journal of Theoretical and Applied Statistics. Vol. 7, No. 5, 2018, pp. $180-187$. \\ doi: $10.11648 /$ j.ajtas.20180705.13
}

Received: July 22, 2018; Accepted: August 7, 2018; Published: September 4, 2018

\begin{abstract}
Bilinear Autoregressive Moving Average Vector (BARMAV) Models are models aggregated with the linear and non-linear vector components of autoregressive and moving average processes. The linear part is the sum of the two vector processes, while the non-linear part is the product of the processes. From the general BARMAV models, Bilinear Autoregressive Vector (BARV) Models and Bilinear Moving Average Vector (BMAV) Models have been isolated. Under certain conditions, the models are proved to exist. Empirically, Nigerian consumer price index and inflation rate are used to test the fitness of the bilinear models. Data for the analysis are from Central Bank of Nigeria Statistical Bulletin, collected from January 2009 to December 2016 with November 2009 as the base year for each of the series. The bilinear autoregressive moving average vector models are fitted to the data. Parameters are tested and found to be significant. The adequacy of each estimated model is confirmed with ACF, PACF and descriptive statistics adopted in the paper. The plots of the actual and fitted CPI and IR have shown that models are adequate as estimates compete favourably with the actual values. The models are useful in modelling some economic and financial data that exhibit some characteristics of non-linearity.
\end{abstract}

Keywords: AR Process, MA Process, Linear and Bilinear Models

\section{Introduction}

When dealing with classical time series models, the two popular processes that explain the behaviour of empirical data in a stationary time series are autoregressive and moving average processes. These processes are described on the basis of autocorrelation and partial autocorrelation functions of empirical data. The popular Autoregressive Moving Average (ARMA) model in time series is a model of linear relationship between a time series process $X_{t}$ and the lag variables of both the process and error term. The General $\operatorname{ARMA}(p, q)$ model is expressed in a linear form as,

$$
X_{t}=\mu_{t}+\sum_{i=1}^{p} \emptyset_{i} X_{t-i}+\sum_{j=1}^{q} \theta_{j} \epsilon_{t-j}+\epsilon_{t}
$$

Where, $X_{t}$ is the time process, $\emptyset_{i}$ and $\theta_{j}$ are parameters of autoregressive and moving average processes respectively, $\epsilon_{t}$ is the error term.

The above model is a univariate linear time series model for the two processes from which AR or MA model can be isolated on condition that $j=0$ or $i=0$ respectively, [2, 5, $8,9]$.

The interest in this paper is to identify special classes of bilinear autoregressive moving average vector models under certain conditions. The fact is that in time series modelling, a process may be described by either AR, MA or both. In as much as AR and MA exist independently each as a univariate linear, multivariate linear, univariate bilinear; it follows that there exists multivariate bilinear model for each of the AR and MA processes under certain conditions.

\subsection{Multivariate ARMA Models}

The multivariate ARMA model is presented in the matrix form as 


$$
\begin{aligned}
& {\left[\begin{array}{c}
x_{1 t} \\
x_{2 t} \\
: \\
\cdot \\
x_{3 t}
\end{array}\right]=\left[\begin{array}{llll}
\alpha_{1.11} & \alpha_{1.12} & \ldots & \alpha_{1.1 c} \\
\alpha_{1.21} & \alpha_{1.22} & \ldots & \alpha_{1.2 c} \\
& \cdot & \cdot & \\
& \cdot & \cdot & \\
\alpha_{1 . r 1} & \alpha_{1 . r 2} & \ldots & \alpha_{1 . r c}
\end{array}\right]\left[\begin{array}{c}
x_{1 t-1} \\
x_{2 t-1} \\
x_{n t-1}
\end{array}\right]+\cdots+\left[\begin{array}{llll}
\alpha_{p .11} & \alpha_{p .12} & \ldots & \alpha_{p .1 c} \\
\alpha_{p .21} & \alpha_{p .22} & \ldots & \alpha_{p .21} \\
& \cdot & \cdot & \\
& \cdot & \cdot & \\
\alpha_{p . r 1} & \alpha_{p . r 2} & \ldots & \alpha_{p . r c}
\end{array}\right]\left[\begin{array}{c}
x_{1 t-p} \\
x_{2 t-p} \\
: \\
\cdot \\
x_{n t-p}
\end{array}\right]} \\
& +\left[\begin{array}{c}
\epsilon_{1 t} \\
\epsilon_{2 t} \\
\vdots \\
\cdot \\
\epsilon_{3 t}
\end{array}\right]+\left[\begin{array}{cccc}
\beta_{1.11} & \beta_{1.12} & \ldots & \beta_{1.1 c} \\
\beta_{1.21} & \beta_{1.22} & \ldots & \beta_{1.2 c} \\
& \cdot & \cdot & \\
& \cdot & \cdot & \\
\beta_{1 . r 1} & \beta_{1 . r 2} & \ldots & \beta_{1 . r c}
\end{array}\right]\left[\begin{array}{l}
\epsilon_{1 t-1} \\
\epsilon_{2 t-1} \\
\epsilon_{n t-1}
\end{array}\right]+\cdots+\left[\begin{array}{llll}
\beta_{p .11} & \beta_{p .12} & \ldots & \beta_{p .1 c} \\
\beta_{p .21} & \beta_{p .22} & \ldots & \beta_{p .21} \\
& \cdot & & \cdot \\
& \cdot & & \cdot \\
\beta_{p . r 1} & \beta_{p . r 2} & \ldots & \beta_{p . r c}
\end{array}\right]\left[\begin{array}{c}
\epsilon_{1 t-p} \\
\epsilon_{2 t-p} \\
: \\
\cdot \\
\epsilon_{n t-p}
\end{array}\right] \\
& X_{i t}=\sum_{a=1}^{p} \sum_{f=1}^{r} \sum_{h=1}^{c} \sum_{j=1}^{n} \alpha_{a . f h} X_{j t-a}+\epsilon_{k t}+\sum_{b=1}^{q} \sum_{f=1}^{r} \sum_{h=1}^{c} \sum_{k=1}^{w} \beta_{b . f h} \epsilon_{k t-b}
\end{aligned}
$$

Model (2) can be written as

$$
X_{i t}=\sum_{a=1}^{p} \sum_{f, h, j=1}^{r c n} \alpha_{a . f h} X_{j t-a}+\epsilon_{k t}+\sum_{b=1}^{q} \sum_{f, h, k=1}^{r c w} \beta_{b . f h} \epsilon_{k t-b}
$$

Where, $X_{i t} \quad(i=1,2, \ldots, n)$ are time series variables, $\epsilon_{k t}(k=1, \ldots, w)$ are error variables associated with $X_{i t}$ with each $i^{\text {th }}$ time process corresponding to each $k^{t h}$ error term $(i=k), X_{j t-a}$ and $\epsilon_{k t-b}$ are autoregressive and moving average processes respectively, $\alpha_{a . f h}(a=$ $1, \ldots, p ; f=1, \ldots r ; h=1, \ldots, c)$ are rxc matrices of autoregressive parameters, $\quad \beta_{b . f h}(b=1, \ldots, q ; f=$ $1, \ldots, r ; h=1, \ldots, c)$ are rxc matrices of moving average parameters. The above model " 3 " is a multivariate linear Autoregressive Moving Average Model. On multivariate time series, cited in this paper includes [17],[6],[11]. From "3" above, if certain condition(s) are introduced to the parameters, each of the processes is isolated; such as $b=0$ changes "3" to Vector Autoregressive (VAR) model in the form,

$$
X_{i t}=\sum_{a=1}^{p} \sum_{f, h, j=1}^{r c n} \alpha_{a . f h} X_{j t-a}+\epsilon_{k t}
$$

Similar to "4" are Dufour [3], Usoro and Omekara [13].

Also if $a=0$, a pure moving average vector model is isolated, and it becomes,

$$
X_{i t}=\sum_{b=1}^{q} \sum_{f, h, k=1}^{r c w} \beta_{b . f h} \epsilon_{k t-b}+\epsilon_{k t}
$$

Where, the parameters are as described above.

\subsection{Non-Linear ARMA Models}

The non-linear Autoregressive Moving Average models are presented in the form,

$$
\begin{aligned}
& {\left[\begin{array}{c}
x_{1 t} \\
x_{2 t} \\
: \\
\cdot \\
x_{3 t}
\end{array}\right]=\left[\begin{array}{cccc}
\varphi_{10.11} & \varphi_{10.12} & \ldots & \varphi_{10.1 c} \\
\varphi_{10.21} & \varphi_{10.22} & \ldots & \varphi_{10.2 c} \\
\cdot & : & \ldots & \cdot \\
\cdot & \cdot & \ldots & \cdot \\
\cdot & \cdot & \cdot \\
\varphi_{10 . r 1} & \varphi_{10 . r 2} & \ldots & \varphi_{10 . r c}
\end{array}\right]\left[\begin{array}{c}
X_{1 t-1} \epsilon_{1 t} \\
X_{2 t-1} \epsilon_{2 t} \\
: \\
\cdot \\
X_{n t-1} \epsilon_{w t}
\end{array}\right]} \\
& \text {. . . . . } \\
& \text {. } . . . \\
& \cdot \begin{array}{ll}
\cdot & \\
. & \ldots
\end{array}
\end{aligned}
$$

$$
\begin{aligned}
& {\left[\begin{array}{cccc}
\varphi_{p 0.11} & \varphi_{p 0.12} & \ldots & \varphi_{p 0.1 c} \\
\varphi_{p 0.21} & \varphi_{p 0.22} & \ldots & \varphi_{p 0.2 c} \\
\vdots & \cdot & \ldots & \vdots \\
\cdot & \cdot & \ldots & \cdot \\
\varphi_{p 0 . r 1} & \varphi_{p 0 . r 2} & \ldots & \varphi_{p 1 . r c}
\end{array}\right]\left[\begin{array}{c}
X_{1 t-p} \epsilon_{1 t} \\
X_{2 t-p} \epsilon_{2 t} \\
\vdots \\
\cdot \\
X_{n t-p} \epsilon_{w t}
\end{array}\right]} \\
& +\left[\begin{array}{cccc}
\varphi_{01.11} & \varphi_{01.12} & \ldots & \varphi_{01.1 c} \\
\varphi_{01.21} & \varphi_{01.22} & \ldots & \varphi_{01.2 c} \\
\cdot & \cdot & \ldots & \vdots \\
\cdot & \cdot & \ldots & \cdot \\
\varphi_{01 . r 1} & \varphi_{01 . r 2} & \ldots & \varphi_{01 . r c}
\end{array}\right]\left[\begin{array}{c}
X_{1 t} \epsilon_{1 t-1} \\
X_{2 t} \epsilon_{2 t-1} \\
: \\
\cdot \\
X_{n t} \epsilon_{w t-1}
\end{array}\right] \\
& {\left[\begin{array}{cccc}
\varphi_{0 Q .11} & \varphi_{0 Q .12} & \cdots & \varphi_{0 Q .1 c} \\
\varphi_{0 Q .21} & \varphi_{0 Q .22} & \ldots & \varphi_{0 Q .2 c} \\
\vdots & \vdots & \cdots & \vdots \\
\cdot & \cdot & \cdots & \cdot \\
\varphi_{0 Q . r 1} & \varphi_{0 Q . r 2} & \cdots & \varphi_{p 0 Q . r c}
\end{array}\right]\left[\begin{array}{c}
X_{1 t} \epsilon_{1 t-Q} \\
X_{2 t} \epsilon_{2 t-Q} \\
\vdots \\
\cdot \\
X_{n t} \epsilon_{w t-Q}
\end{array}\right]}
\end{aligned}
$$

The expansion of the above matrices gives non-linear ARMA models for $X_{1 t}, X_{2 t}, \ldots, X_{n t}$. The models are reduced to

$X_{i t}=\sum_{a=1}^{P} \sum_{b=1}^{Q} \sum_{f=1}^{r} \sum_{h=1}^{c} \sum_{j=1}^{n} \sum_{k=1}^{w} \varphi_{a b . f h} X_{j t-a} \epsilon_{k t-b}+\epsilon_{k t}(6)$

Model (6) can be written as

$$
X_{i t}=\sum_{a=1}^{P} \sum_{b=1}^{Q} \sum_{f, h, j, k=1}^{r c n w} \varphi_{a b . f h} X_{j t-a} \epsilon_{k t-b}+\epsilon_{k t}(7)
$$

Model "7" is a multivariate non-linear Autoregressive Moving Average Model.

Combining " 5 " and "7", we have Multivariate Bilinear Autoregressive Moving Average Models also known as BARMAV models as 


$$
\begin{aligned}
X_{i t} & =\sum_{a=1}^{p} \sum_{f, h, j=1}^{r c n} \alpha_{a . f h} X_{j t-a}+\sum_{b=1}^{q} \sum_{f, h, k=1}^{r c w} \beta_{b . f h} \epsilon_{k t-b} \\
& +\sum_{a=1}^{P} \sum_{b=1}^{Q} \sum_{f, h, j, k=1}^{r c n w} \varphi_{a b . f h} X_{j t-a} \epsilon_{k t-b}+\epsilon_{k t}
\end{aligned}
$$

Where $\alpha_{a . f h}, \beta_{b . f h}, \varphi_{a b . f h} \neq 0$ and $1 \leq a \leq p, P ; 1 \leq b \leq$ $q, Q ; 1 \leq j \leq n ; 1 \leq k \leq w ; 1 \leq f, h, \leq r, c$.

Model " 8 " is the multivariate form of the univariate models presented by Granger and Anderson [4], Maravall [10], Subba Rao and Gabr [12], Bibi and Oyet [1], Iwueze $[7]$.

$$
X_{i t}=\sum_{a=1}^{p} \sum_{f, h, j=1}^{r c n} \alpha_{a . f h} X_{j t-a}+\sum_{a=1}^{P} \sum_{f, h, j, k=1}^{r c n w} \varphi_{a 0 . f h} X_{j t-a} \epsilon_{k t}+\epsilon_{k t}
$$

Proof:

From "8",

$$
\text { Let } X_{i t}=A_{t}+B_{t}+C_{t}+\epsilon_{k t}
$$

Where,

$$
A_{t}=\sum_{a=1}^{p} \sum_{f, h, j=1}^{r c n} \alpha_{a . f h} X_{j t-a}, B_{t}=\sum_{b=1}^{q} \sum_{f, h, k=1}^{r c w} \beta_{b . f h} \epsilon_{k t-b}
$$

and

$$
C_{t}=\sum_{a=1}^{P} \sum_{b=1}^{Q} \sum_{f, h, j, k=1}^{r c n w} \varphi_{a b . f h} X_{j t-a} \epsilon_{k t-b}
$$

Special Condition: if $\mathrm{b}=0 \Rightarrow \mathrm{q}=0, \beta_{0 . f h}(f=1, \ldots, r ; h=1, \ldots, c)=0$, the moving average component of the model is uncorrelated at any lag, $=>B_{t}=0$, as $\epsilon_{k t} \sim \operatorname{iid}\left(0, \sigma_{e}^{2}\right)$,

then

$$
\begin{gathered}
A_{t}=\sum_{a=1}^{p} \sum_{f, h, j=1}^{r c n} \alpha_{a . f h} X_{j t-a} \text { and } C=\sum_{a=1}^{P} \sum_{f, h, j, k=1}^{r c n w} \varphi_{a 0 . f h} X_{j t-a} \epsilon_{k t}, \text { such that } \\
X_{i t}=A_{t}+C_{t}+\epsilon_{k t}=\sum_{a=1}^{p} \sum_{f, h, j=1}^{r c n} \alpha_{a . f h} X_{j t-a}+\sum_{a=1}^{P} \sum_{f, h, j, k=1}^{r c n w} \varphi_{a 0 . f h} X_{j t-a} \epsilon_{k t}+\epsilon_{k t}
\end{gathered}
$$

Hence, "9" is BARV model with $\alpha_{a . f h}, 1 \leq a \leq p, 1 \leq f, h, j \leq r, c, n ; \varphi_{a 0 . f h}, 1 \leq a \leq P, 1 \leq f, h, j, k \leq r, c, n, w$. This completes the proof.

\subsection{Bilinear Moving Average Vector (BMAV) Model}

BMAV model is given as

$$
X_{i t}=\sum_{b=1}^{q} \sum_{f, h, k=1}^{r c w} \beta_{b . f h} \epsilon_{k t-b}+\sum_{b=1}^{Q} \sum_{f, h, j, k=1}^{r c n w} \varphi_{0 b . f h} X_{j t} \epsilon_{k t-b}+\epsilon_{k t}
$$

Corollary

From "8"

$$
\text { Let } X_{i t}=D_{t}+E_{t}+F_{t}+\epsilon_{k t}
$$

Where,

$$
D_{t}=\sum_{a=1}^{p} \sum_{f, h, j=1}^{r c n} \alpha_{a . f h} X_{j t-a}, E_{t}=\sum_{b=1}^{q} \sum_{f, h, k=1}^{r c w} \beta_{b . f h} \epsilon_{k t-b}
$$


and

$$
F_{t}=\sum_{a=1}^{P} \sum_{b=1}^{Q} \sum_{f, h, j, k=1}^{r c n w} \varphi_{a b . f h} X_{j t-a} \epsilon_{k t-b}
$$

Special Condition: if $\mathrm{a}=0 \Rightarrow \mathrm{p}=0, \alpha_{0 . f h}(f=1, \ldots, r ; h=1, \ldots, c)=0$, the autoregressive component of the model is uncorrelated at any lag, $=>D_{t}=0$, as $\epsilon_{k t} \sim \operatorname{iid}\left(0, \sigma_{e}^{2}\right)$,

then

$$
\begin{gathered}
E_{t}=\sum_{b=1}^{q} \sum_{f, h, j=1}^{r c w} \beta_{b . f h} \epsilon_{k t-b} \text { and } F_{t}=\sum_{b=1}^{Q} \sum_{f, h, j, k=1}^{r c n w} \varphi_{0 b . f h} X_{j t} \epsilon_{k t-b}, \text { such that } \\
X_{i t}=E_{t}+F_{t}+\epsilon_{k t}=\sum_{b=1}^{q} \sum_{f, h, j=1}^{r c w} \beta_{b . f h} \epsilon_{k t-b}+\sum_{b=1}^{Q} \sum_{f, h, j, k=1}^{r c n w} \varphi_{0 b . f h} X_{j t} \epsilon_{k t-b}+\epsilon_{k t}
\end{gathered}
$$

Hence, "10" is BMAV model with $\beta_{b . f h}, 1 \leq b \leq q, 1 \leq$ $f, h, j \leq r, c, w ; \varphi_{0 b . f h}, 1 \leq b \leq Q, 1 \leq f, h, j, k \leq$ $r, c, n, w$. This completes the proof.

\subsection{Test for Linearity and Bilinearity}

The above test involves the parameters of both the linear and non-linear components of bilinear time series models.

$$
\begin{gathered}
t=\frac{\hat{\alpha}_{a . f h}}{s_{\widehat{\alpha}_{a . f h}}} \text { for the linear AR component }, \frac{\hat{\beta}_{b . f h}}{s_{\widehat{\beta}_{b . f h}}} \text { for the linear MA component, } \\
\text { and } \frac{\hat{\varphi}_{a b . f h}}{s_{\widehat{\varphi}_{a b . f h}}} \text { for the non - linear ARMA component. }
\end{gathered}
$$

Hypotheses:

$\mathrm{H}_{0}: \hat{\alpha}_{a . f h}=\hat{\beta}_{b . f h}=\hat{\varphi}_{a b . f h}=0$

$\mathrm{H}_{1}: \hat{\alpha}_{a . f h} \neq 0, \hat{\beta}_{b . f h} \neq 0, \hat{\varphi}_{a b . f h} \neq 0$

From the above hypotheses;

(i) if $\mathrm{H}_{0}$ is rejected for at least one of $\hat{\alpha}_{a . f h}$ and $\hat{\beta}_{b . f h}$, but accepted for $\hat{\varphi}_{a b . f h}$, there is no effect of bilinearity in the series. It is characterised by a pure linear process.

(ii) if $\mathrm{H}_{0}$ is accepted for both $\hat{\alpha}_{a . f h}$ and $\hat{\beta}_{b . f h}$, but rejected for only $\hat{\varphi}_{a b . f h}$, the series is characterised by a complete non-linear process.

(iii) if $\mathrm{H}_{0}$ is rejected for at least one of $\hat{\alpha}_{a . f h}$ and $\hat{\beta}_{b . f h}$, and rejected for $\hat{\varphi}_{a b . f h}$, there is effect of bilinearity in the series. A bilinear process is a process that has effects of both linear and nonlinear components in the series. For a time series exhibits bilinear characteristics only if at least a parameter of a linear component is significant with the parameter of the nonlinear component of the model.

\section{Model Fitting to Empirical Data}

In this section, we consider fitting the bilinear models to (i) Linear Parameter: A linear parameter is the unknown coefficient of either AR, MA or Mixed ARMA process.

(ii) Non-Linear Parameter: This is the unknown coefficient of the non-linear component(s) of the bilinear model.

Test Statistic:

The test statistic is given as the empirical data. For illustration, Nigeria Consumer Price Index (CPI) and Inflation rates (IR) are fitted with the bilinear models. The procedures of fitting bilinear models to time series data are not different from the ordinary ARMA models.

\subsection{Time Plots of the CPI and IR}

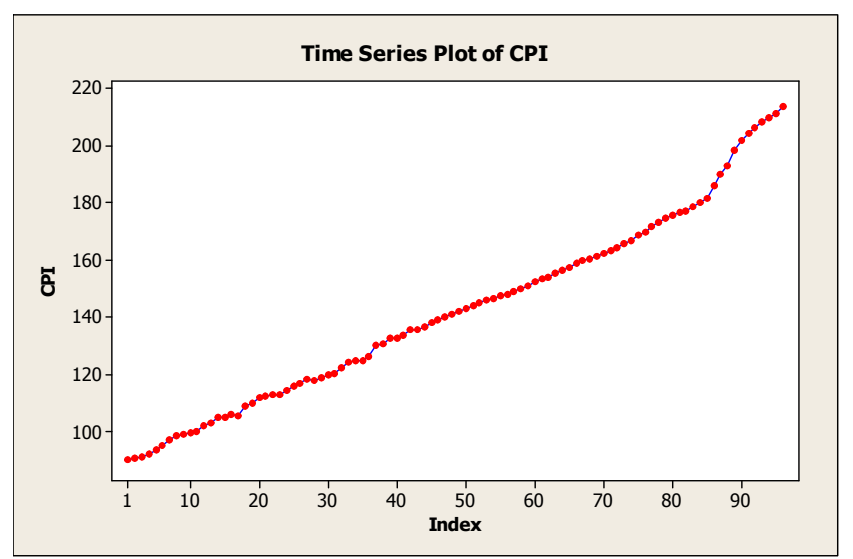

Figure 1. Time Series Plot of Consumer Price Index. 


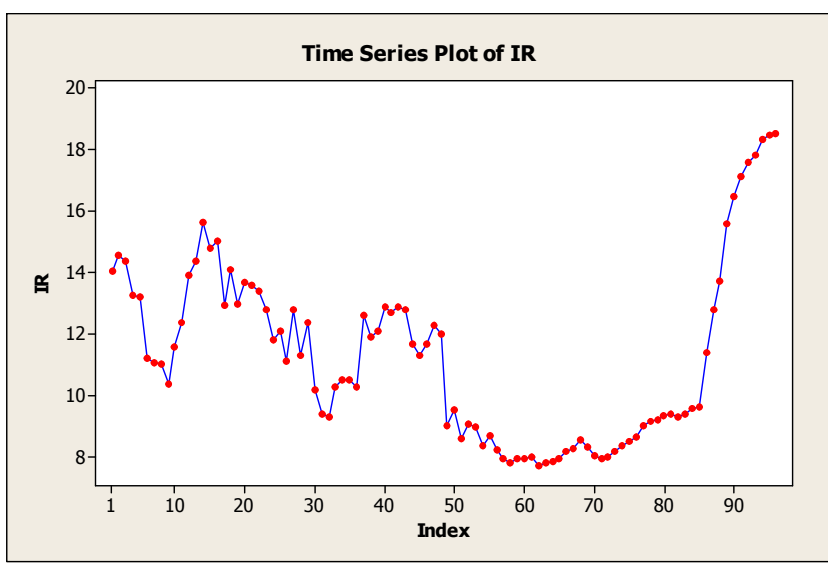

Figure 2. Time Series Plot of Inflation Rate.

"Figures 1" and "Figure 2" are the time series plots of consumer price index and inflation rates from January 2009 to December 2016, with November 2009 as the base year. The consumer price index exhibits long term increase over time, while interest rate shows some level of randomness, which explains non-linearity characteristics as indicated from January 2009 with a drop 2013, and sharp increase in 2016.

\subsection{Autocorrelation and Partial Autocorrelation Functions of CPI and IR}

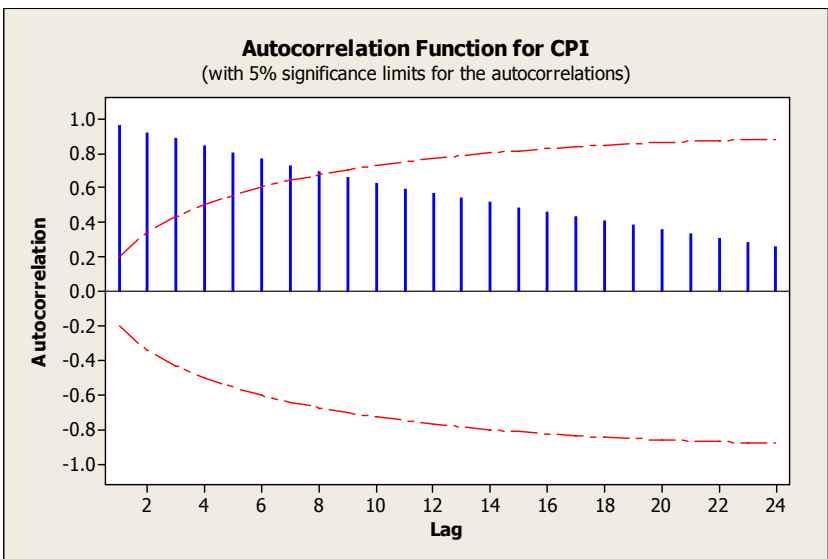

Figure 3. $A C F$ of the $C P I$.

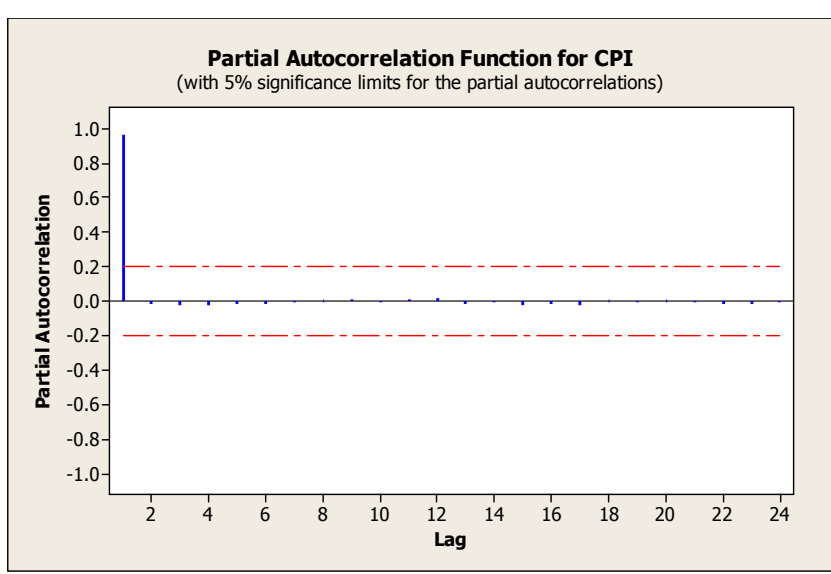

Figure 4. PACF of the CPI.

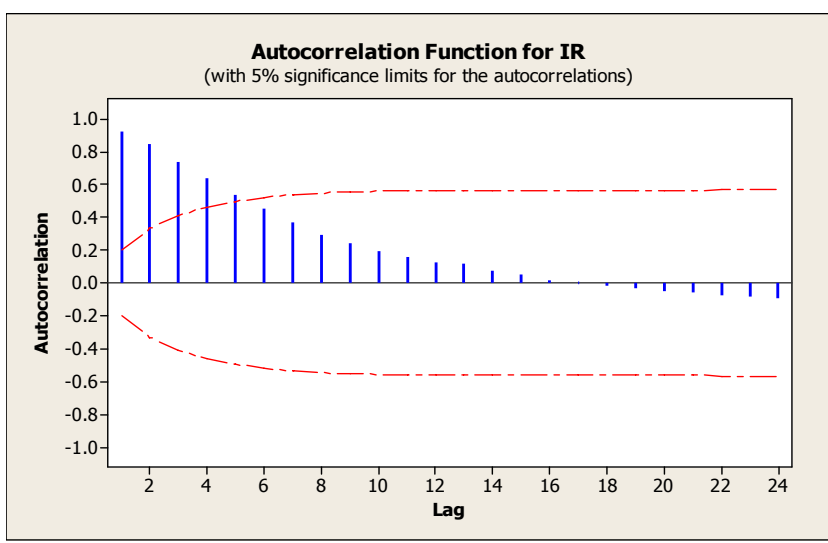

Figure 5. ACF of IR.

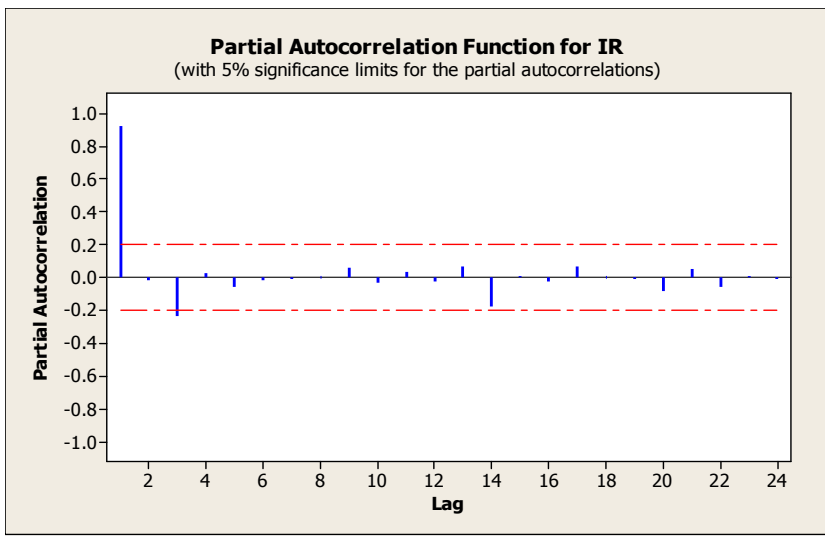

Figure 6. PACF of IR.

The above ACF's and PACF's for the CPI and IR suggest $\operatorname{BARMA}(1,1,1,1)$ for each CPI and IR.

\subsection{Estimation of Parameters and Interpretation of Results}

Table 1. Estimation of Model Parameters for $C P I\left(X_{l t}\right)$.

\begin{tabular}{lllll}
\hline Predictor & Coeff. & SE. Coeff & t-statistics & P \\
\hline Constant & 0.9864 & 0.7978 & 1.24 & 0.220 \\
$\mathrm{X}_{1 \mathrm{t}-1}$ & 0.9972 & 0.0042 & 240.12 & 0.000 \\
$\mathrm{X}_{2 \mathrm{t}-1}$ & 0.01896 & 0.0328 & 0.58 & 0.564 \\
$\epsilon_{1 t-1}$ & -0.8520 & 0.3779 & 2.25 & 0.027 \\
$\epsilon_{2 t-1}$ & 1.2686 & 0.6063 & 2.09 & 0.039 \\
$\mathrm{X}_{1 \mathrm{t}-1} \epsilon_{1 t-1}$ & 0.0093 & 0.0024 & 3.92 & 0.000 \\
$\mathrm{X}_{2 \mathrm{t}-1} \epsilon_{2 t-1}$ & -0.1412 & 0.0533 & 2.65 & 0.009 \\
\hline
\end{tabular}

Table 2. Estimation of Model Parameters for $\operatorname{IR}\left(X_{2 t}\right)$.

\begin{tabular}{lllll}
\hline Predictor & Coeff. & SE. Coeff & t-statistics & P \\
\hline Constant & 1.1815 & 0.8569 & 1.38 & 0.171 \\
$\mathrm{X}_{1 \mathrm{t}-1}$ & -0.0058 & 0.0045 & -1.30 & 0.196 \\
$\mathrm{X}_{2 \mathrm{t}-1}$ & 0.9529 & 0.0352 & 27.08 & 0.000 \\
$\epsilon_{1 t-1}$ & -1.0104 & 0.4058 & -2.49 & 0.015 \\
$\epsilon_{2 t-1}$ & 0.1491 & 0.6512 & 0.23 & 0.819 \\
$\mathrm{X}_{1 \mathrm{t}-1} \epsilon_{1 t-1}$ & 0.0082 & 0.0026 & 3.20 & 0.002 \\
$\mathrm{X}_{2 \mathrm{t}-1} \epsilon_{2 t-1}$ & -0.0308 & 0.0572 & -0.54 & 0.592 \\
\hline
\end{tabular}

The models with parameter estimates are presented as follows 


$$
\begin{array}{r}
{\left[\begin{array}{l}
X_{1 t} \\
X_{2 t}
\end{array}\right]=\left[\begin{array}{cc}
1.00183 & 0.04905 \\
-0.000286 & 0.98898
\end{array}\right]\left[\begin{array}{l}
X_{1 t-1} \\
X_{2 t-1}
\end{array}\right]} \\
+\left[\begin{array}{ll}
-0.5241 & 1.3913 \\
-0.6176 & 0.2961
\end{array}\right]\left[\begin{array}{c}
\epsilon_{1 t-1} \\
X \epsilon_{2 t-1}
\end{array}\right] \\
+\left[\begin{array}{ll}
0.007128 & -0.15476 \\
0.005565 & -0.04692
\end{array}\right]\left[\begin{array}{l}
X_{1 t-1} \epsilon_{1 t-1} \\
X_{2 t-1} \epsilon_{2 t-1}
\end{array}\right]
\end{array}
$$

The above results have it that parameters of the bilinear model fitted to CPI are all significant, except for $\mathrm{X}_{2 \mathrm{t}-1 \text {. }}$ For IR, evidence has it that some parameters of the linear components are significant with one of the non-linear components. This is a true indication of model fitness to the data. Further evidence is shown in the ACF and PACF of the model residual.

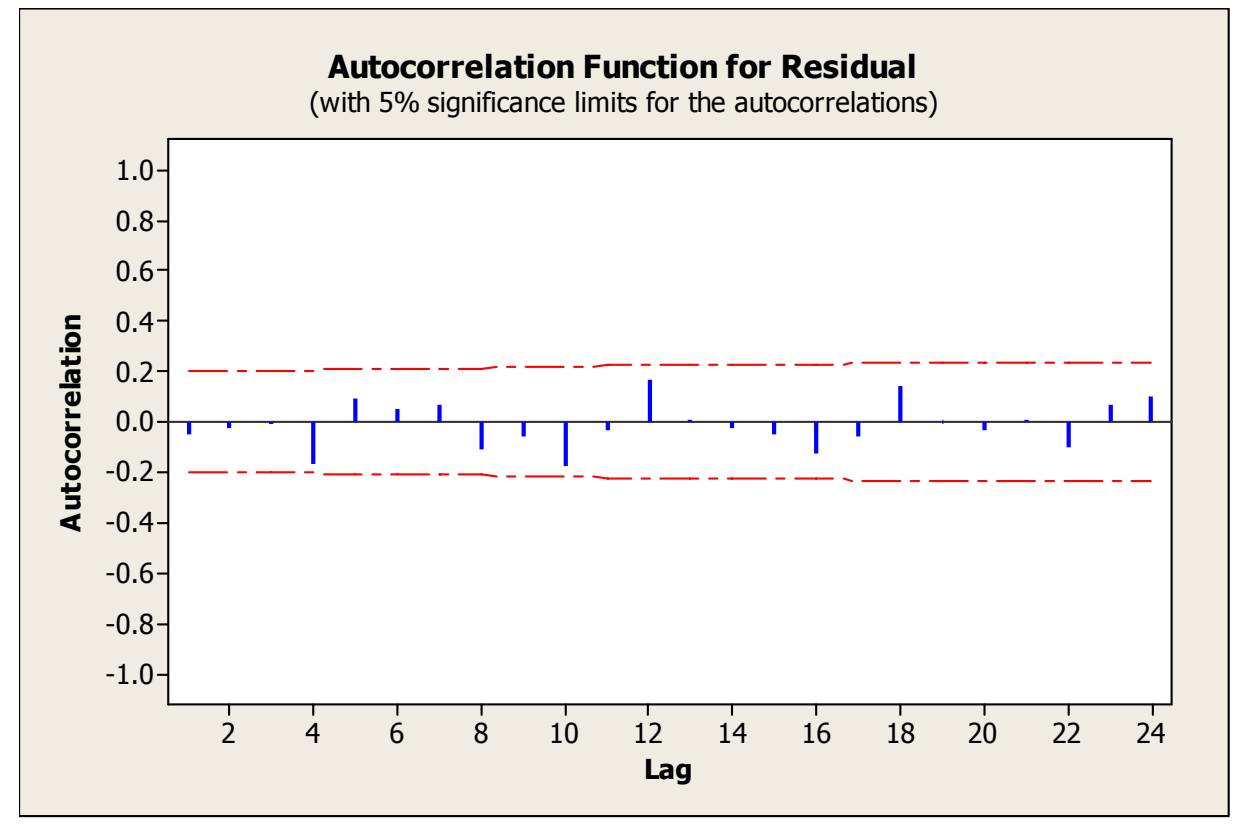

Figure 7. ACF of the Model Residual.

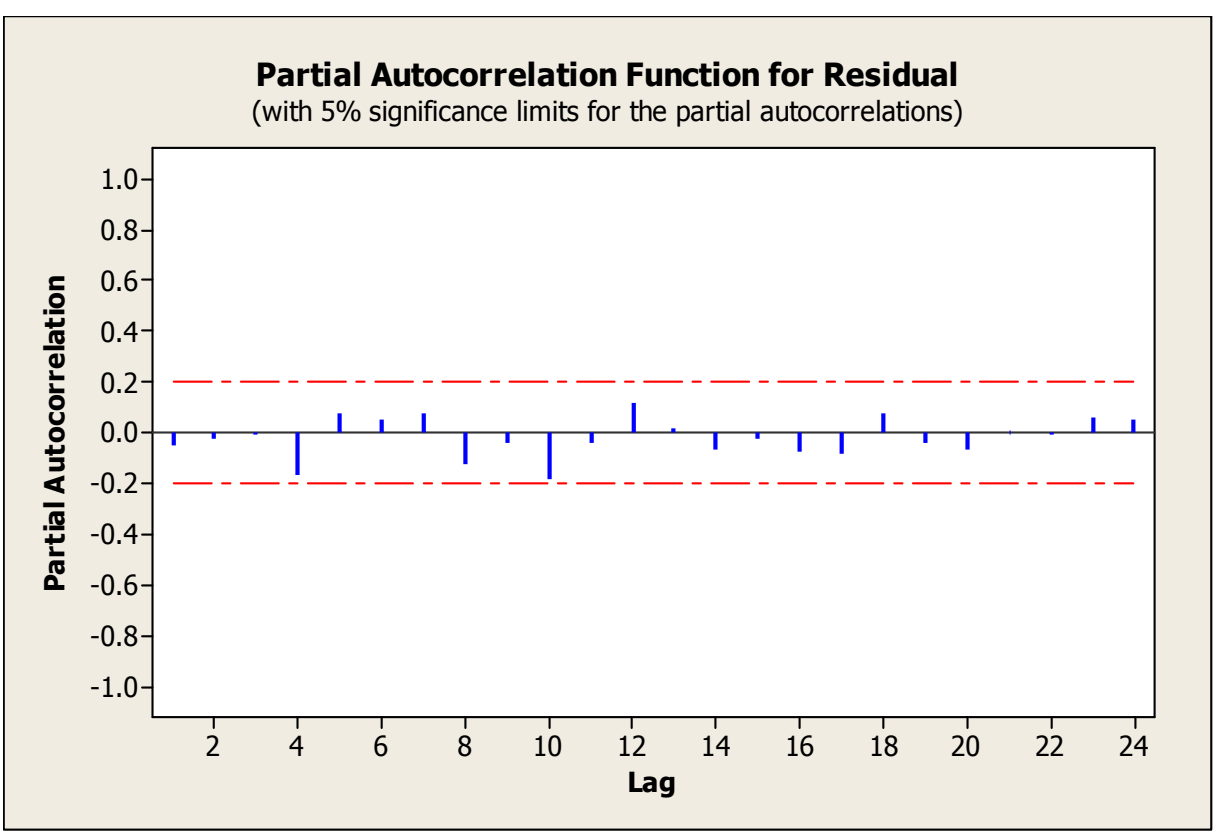

Figure 8. PACF of the Model Residual.

"Figure 7" and "Figure 8" are evident that the residual is a pure white noise process, which is identically and independently

\begin{tabular}{|c|c|c|c|c|c|c|c|c|c|c|}
\hline Variable & $\mathbf{N}$ & $\mathbf{N}^{*}$ & Mean & SE Mean & STD & Min & Q1 & Median & Q3 & Max \\
\hline Residual & 95 & 1 & 0.000 & 0.073 & 0.708 & -1.3 & -0.3 & -0.11 & 0.23 & 3.0 \\
\hline
\end{tabular}
distributed with zero mean and constant variance as shown in table3.

Table 3. Descriptive Statistics: Residual. 


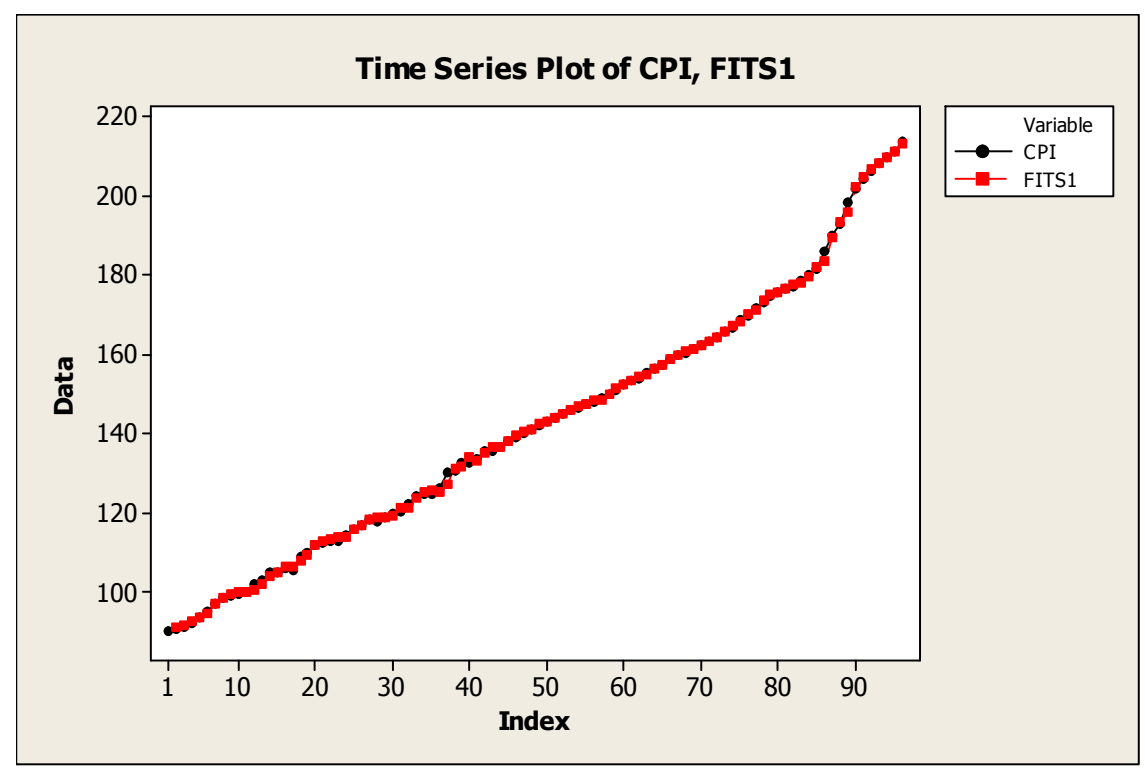

Figure 9. Plots of Actual and Fitted CPI Series.

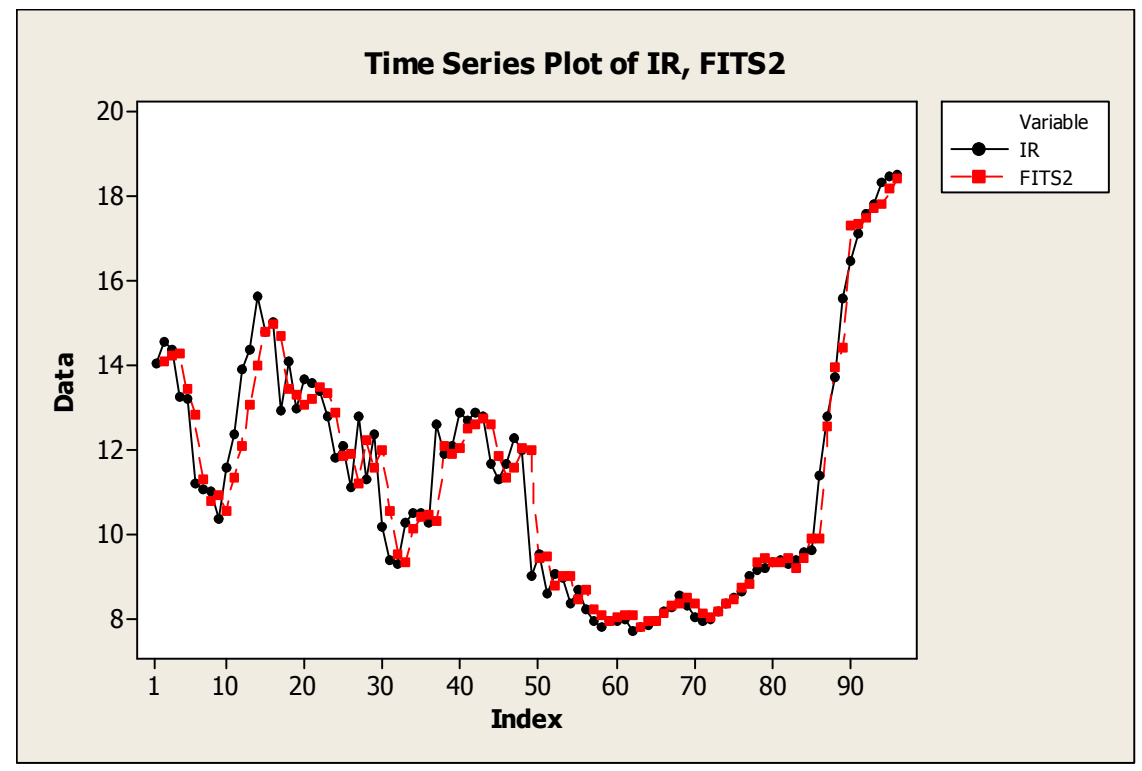

Figure 10. Plots of Actual and Fitted IR Series.

\section{Conclusion}

There is no gainsaying the fact that each of the AR and MA processes are combined to form mixed ARMA process. This is explained by the behaviour of the empirical data as always shown in the distribution of the autocorrelation and partial autocorrelation functions. A process that is only described by either AR or MA remains a singular process, except characterised by both. The idea about this paper is that if there exist condition(s) for isolation of AR or MA from ARMA, isolation of BAR or BMA from BARMA models, therefore, BARV and BMAV are conditionally isolated from BARMAV model. For a pure bilinear autoregressive vector model, the non-linear component of the model is the product of lagged $X_{i t}$ and non-lagged $\epsilon_{k t}$. That is the multiplication of $X_{i t-1}, X_{i t-2}, \ldots, X_{i t-P}$ by $\epsilon_{k t}$. That means each of the non- zero lags of $X_{i t}$ is multiplied by zero lag of $\epsilon_{k t}$ to form the non-linear part of the model. Similarly, for a pure bilinear moving average vector model, the non-linear component of the model is the product of lagged $\epsilon_{k t}$ and non-lagged $X_{i t}$. That is the multiplication of $\epsilon_{k t-1}, \ldots, \epsilon_{k t-Q}$ by $X_{i t}$. Here, each of the non-zero lags of $\epsilon_{k t}$ is multiplied by zero lag of $X_{i t}$ to form the non-linear part of the BMAV model. Empirically, see Usoro and Omekara (2008) and Usoro (2018). Empirically, the monthly consumer price index and inflation rate used in this paper are characterised by both AR and MA process. This called for adoption of " 8 " in the analysis. The plots of the actual and fitted CPI and IR data in figures "9" and "10" have shown that estimates compete favourably with the actual. Hence, the models are suitable in modelling time series data that exhibit some form of nonlinearity characteristics. 


\section{References}

[1] Bibi, A. and Oyet, A. J. (1991): Estimation of some bilinear time series models with time varying coefficients, AMS.

[2] Box, G. P. and Jenkins, G. M (1978): Time Series Analysis, Forecasting and Control. Holden-Day, San Francisco.

[3] Dufour Jean-Marie (2006): Multivariate Time Series Modelling. Cambridge University Press, Cambridge, U.K.

[4] Granger, C. W. J. and Anderson, A. P (1978): Introduction to Bilinear Time Series Models. Vandenhoeck and Ruprecht.

[5] Gujarati, Damodar N.and Porter, Dawn C. (2009): Basic Econometrics, Fifth Edition.

[6] Harrison L., Penny, W. D. and Friston, K. J. (2003): Application of multivariate time series in the functional network in the brain region. Neuroimage, 19(4), 1477-1491.

[7] Iwueze S. I. (2002): Vectorial representation and its application to covariance analysis of super-diagonal bilinear time series models. The Physical Scientist 1(1), 85-96.

[8] Johnston Jack and DiNardo John (1997): Econometric Methods. International Edition.

[9] Kendell M. and Ord Keith J. (1990): Time Series. Third Edition, Halsted Press, Third Avenue, New York.

[10] Maravall, A. (1983): An application of non-linear time series forecasting. Journal of Business and Economic Statistics Vol. $1(1), 66-74$.

[11] Sims, C. A. (1996): Multivariate Time Series Modelling of Gross National Products of United State of America. American Statistical Association Meetings.

[12] Subba Rao, T. and Gabr, M. M. (1984): An introduction to bispectral analysis and bilinear time series models. Lecture Notes in Statistics No.24. Springer Verlag.

[13] Usoro, A. E. and Omekara, C. O. (2007): Estimation of Pure Autoregressive Models for Revenue Series. Global Journal of Mathematical Sciences, 6(1), 31-37.

[14] Usoro, A. E. and Omekara C. O. (2008): Bilinear autoregressive vector models and their applications to estimation of revenue series. Asian Journal of Mathematics and Statistics 1(1): 50-56.

[15] Usoro, Anthony E. (2017): Identification of Classes of Bilinear Time Series Models. Journal of Statistics; Advances in Theory and Applications. Vol.17, No.2. 153-160.

[16] Usoro, Anthony E. (2018): Modelling of Nigerian gross domestic product using seasonal and bilinear autoregressive integrated moving average models. Journal of Statistical and Econometric Methods, Vol.7, Issue 2.

[17] Wei, W. S. (1990): Time Series Analysis; Univariate and Multivariate Methods. California, Addison-Wesley. 